\title{
PHOTOCATALYTIC REMOVAL OF TRI- AND HEXA-VALENT CHROMIUM IONS FROM CHROME-ELECTROPLATING WASTEWATER
}

\author{
Puangrat Kajitvichyanukul $^{* 1,2}$ and Chulaluck Changul ${ }^{1}$ \\ ${ }^{1}$ Department of Environmental Engineering, \\ King Mongkut's University of Technology Thonburi, Bangkok, Thailand \\ ${ }^{2}$ National Research Center for Environmental and Hazardous Waste Management, \\ Chulalongkorn University, Bangkok, Thailand
}

Received 04 July 2005

\begin{abstract}
A novel technique based on photocatalysis was applied to eliminate chromium ions, a toxic hazardous environmental pollutant. The photoreduction of each species of chromium (total, hexavalent, and trivalent chromiums) from chrome-electroplating wastewater was investigated using a titanium dioxide suspension under irradiation by a low-pressure mercury lamp. The initial concentration of total chromium was $300 \mathrm{mg} / \mathrm{l}$. The applied conditions were the direct photocatalytic reduction process at $\mathrm{pH} 3.65$ and the indirect photocatalytic reduction with added hole scavengers at the same solution $\mathrm{pH}$. Results from both processes were comparatively discussed. Result show that chromium was not efficiently removed by direct photoreduction. In contrast, with the adding of hole scavengers, which were formate ions, the photoreduction of chromium was very favorable. Both hexavalent and trivalent chromiums were efficiently removed. The photocatalytic mechanism is purposed in this study.
\end{abstract}

\section{INTRODUCTION}

Recently, a novel technology based on a photocatalytic process in aqueous suspensions of semiconductors, has received considerable attention in studies on the removal of hazardous waste from water and wastewater $[1,4,7]$. Upon illumination of the semiconductor-electrolyte interface with light of energy higher than the semiconductor band gap, electron-hole pairs $\left(\mathrm{e}^{-}-\mathrm{h}^{+}\right)$ are formed in the conduction and valence bands of the semiconductor, respectively. These charge carriers, which migrate to the semiconductor surface, are capable of reducing or oxidizing pollutant species in solution, having suitable redox potentials. Many semiconductors and metal oxides, such as $\mathrm{TiO}_{2}, \mathrm{ZnO}, \mathrm{SiO}_{2}, \mathrm{Al}_{2} \mathrm{O}_{3}$ and $\mathrm{Fe}_{2} \mathrm{O}_{3}$, have been used as catalysts for the photocatalytic process. Titanium dioxide $\left(\mathrm{TiO}_{2}\right)$ is an effective catalyst and is often used for the photocatalysis process owing to its optical and electronic properties, chemical stability and nontoxicity for the environment [11]. More recently, an interesting technological application of this

*Corresponding author e-mail: puangrat.kaj@kmutt.ac.th 
photocatalysis has been applied for eliminating toxic metals (chromium, thallium, mercury, lead, among others) from water and wastewater [1, 4].

This paper describes results from a study on the removal of chromium from chromeelectroplating wastewater using this technology. The most stable oxidation states of chromium in the environment are chromium(III) and chromium(VI). The concentration and toxicity of soluble chromium and its mobility in aquatic and terrestrial environments depend on its oxidation state. Chromium(VI) is more toxic and generally more mobile than Chromium(III). Conventional methods for treatment of contaminated Chromium(VI) include chemical reduction, ion exchange and adsorption on coal or activated carbon. However, most of these methods require either high energy or large quantities of chemicals, and the photocatalytic process has been found to be superior [9].

Our approach in this study differs from that of previous authors [2, 5, 8, 9], in that the removal of chromium is from real wastewater while the prior works focused mainly on the removal of chromium(VI) from synthetic waste. The constituents in real wastewater are much more complicated than those from synthetic wastewater, thus, different results might be expected. The results and efficiencies of the employed photocatalytic process in each system (direct and indirect photoreduction) are described here.

\section{EXPERIMENTAL}

All chemicals were reagent grade or better and were used as received. The $\mathrm{TiO}_{2}$ (Degussa P-25) was predominantly anatase and had a specific surface area of $\sim 60 \mathrm{~m}^{2} \mathrm{~g}^{-1}$. Figure 1 shows a schematic of the photoreactor, which was employed in this study. A $10 \mathrm{~W}$ low-pressure Hg UVlamp was used as the radiation source. The quartz reactor consisted of two compartments: the outer for containing wastewater and the inner for housing a UV lamp. The inner compartment was water-jacketed to maintain the solution temperature at 25 degrees Celsius for all tests, and equipped with a magnetic stirrer.

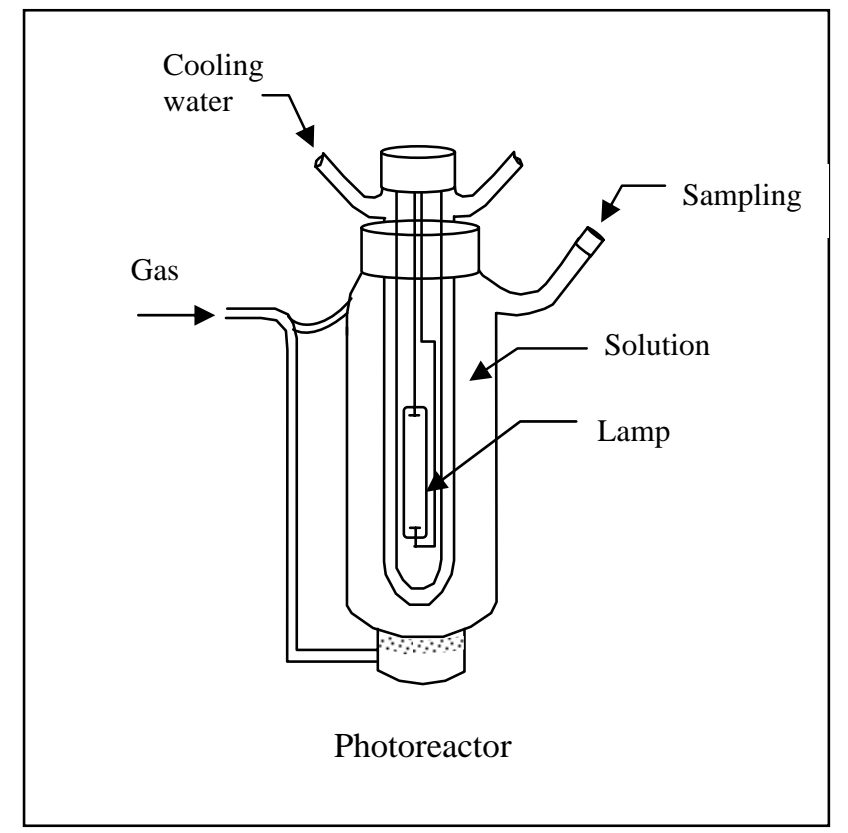

Fig. 1: $\quad$ A schematic of the photoreactor employed in this study 
The concentration of $\mathrm{TiO}_{2}$ was nominally $20 \mathrm{~g} / \mathrm{L}$, unless otherwise specified. Characteristic of the chrome-wastewater used in this study are shown in Table 1. For comparison, the photocatalytic process was applied to chromium from synthetic wastewater using $\mathrm{Cr}_{2}\left(\mathrm{SO}_{4}\right)_{3}$ and $\mathrm{K}_{2} \mathrm{Cr}_{2} \mathrm{O}_{7}$ as sources for trivalent and hexavalent chromium species, respectively. The wastewater loaded $\mathrm{TiO}_{2}$ suspensions were equilibrated in the dark for 15 minutes. After the equilibration period, the ultraviolet light (UV) was turned on and the solution was syringed out periodically. The solution was sampled at various times during each experiment by withdrawing milliliters of aliquots and removing the suspended catalyst by filtration. All the samples were covered by aluminum paper to avoid sunlight illumination. The solution $\mathrm{pH}$ was adjusted using $\mathrm{NaOH}$ (basic range) and $\mathrm{H}_{2} \mathrm{SO}_{4}$ (acidic range). All experiments were accomplished at normal temperature $\left(25^{\circ} \mathrm{C} \pm 2^{\circ} \mathrm{C}\right)$.

All the solutions were tested for remaining concentrations of metals ions by a colorimetric method. UV-Visible spectra for chromium(VI) measurements were recorded on a HewlettPackard model diode array spectrometer.

Total chromium concentration in supernatant solution was determined using Inductive Couple Plasma Spectrometer, ICP (made by Perkin Elmer, model Optima 2000 DV). The chromium(VI) concentration was determined using the diphenylcarbazide colorimetric method in which stoichiometric oxidation of the dipenylcarbazide reagent yielded a product with an absorption peak at $540 \mathrm{~nm}$. The chromium(III) concentration was determined by subtraction of chromium(VI) from the total chromium concentration.

Table 1: $\quad$ Characteristics of chrome-wastewater used in this study

\begin{tabular}{ll}
\hline $\mathrm{pH}$ & 3.65 \\
Total solid, mg/l & 2750 \\
Total dissolved solid, mg/l & 2150 \\
Total chromium, mg/l & 300 \\
Hexavalent chromium, mg/l & 150 \\
Trivalent chromium, mg/l & 150 \\
Sulphate, mg/l & 1150 \\
Chloride, mg/l & 127.5 \\
Nitrate, mg/l & 292 \\
COD, mg/l & Nil \\
\hline
\end{tabular}

\section{RESULTS AND DISCUSSION}

\subsection{Dark adsorption of chromium on titanium dioxide surface}

Chromium adsorption onto different amounts of titanium dioxide was examined as a function of time at original $\mathrm{pH}$ to obtain the equilibration time prior to irradiation. As shown in previous study [5], the optimum $\mathrm{pH}$ for chromium photoreduction is at $\mathrm{pH} 3$. Thus, the solution $\mathrm{pH}$ of this study did not require any adjusting. It can be seen that total chromium reached the highest amount of adsorption onto the $\mathrm{TiO}_{2}$ surface within 15 minutes for all conditions applied in this experiment set (Fig. 2). The amount of adsorbed chromium was directly proportionate with the provided $\mathrm{TiO}_{2}$ in the system. The fact that a number of adsorbed surface sites are increased with the amount of $\mathrm{TiO}_{2}$ might be the best explanation in this concern.

From our earlier studies, [6] and those of other groups [3, 8], the maximum adsorption of chromium(VI) onto $\mathrm{TiO}_{2}$ surface in the dark was found at $\mathrm{pH} 3-4$. As the $\mathrm{pH}$ decreased or increased from this region, the chromium(VI) adsorption was reduced. Particularly, chromium(III) exists as $\mathrm{Cr}^{3+}$ in solution and as "cation-like" species. It is known that chromium(III) adsorption increases as the $\mathrm{pH}$ is increased [3]. 


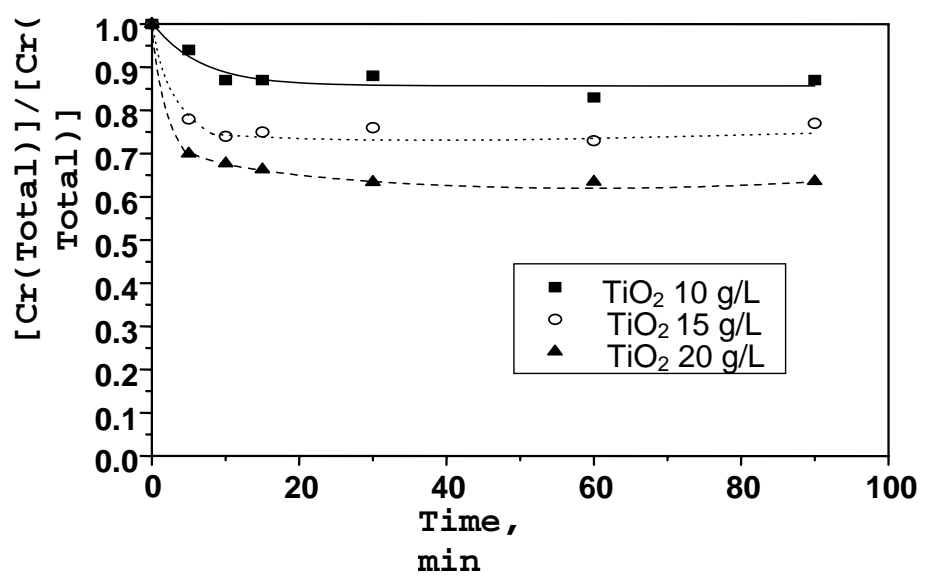

Fig. 2: $\quad$ The equilibrium adsorption of total chromium onto $\mathrm{TiO}_{2}$ surface at $\mathrm{pH} 3.65$

\subsection{Direct photoreduction of chromium on titanium dioxide surface}

Figure 3 shows the results of total chromium reduction after 30 minutes adsorption and 180 minutes irradiation periods. A series of experiments was conducted to investigate the removal efficiency of chromium(VI) by different amounts of $\mathrm{TiO}_{2}$. Recall that the value at 30 minutes refers to amounts of chromium at the end of the dark equilibration, which reflects the chromium removal by adsorption during the equilibration period.

As seen from the graph, total chromium reduced remarkably with different amounts of $\mathrm{TiO}_{2}$ particles in the solution. The reduction of chromium rapidly decreased during the first 90 minutes of experiment. Beyond that, there was no change in chromium concentration. It may be presumed that the surface of $\mathrm{TiO}_{2}$ is fully covered by chromium in the first period. Apparently, higher amounts of $\mathrm{TiO}_{2}$ were required to complete photoreduction of total chromium. However, it is worth noting that the excess $\mathrm{TiO}_{2}$ caused a reduction in chromium removal efficiency due to the inhibition of light scattering by $\mathrm{TiO}_{2}$ itself.

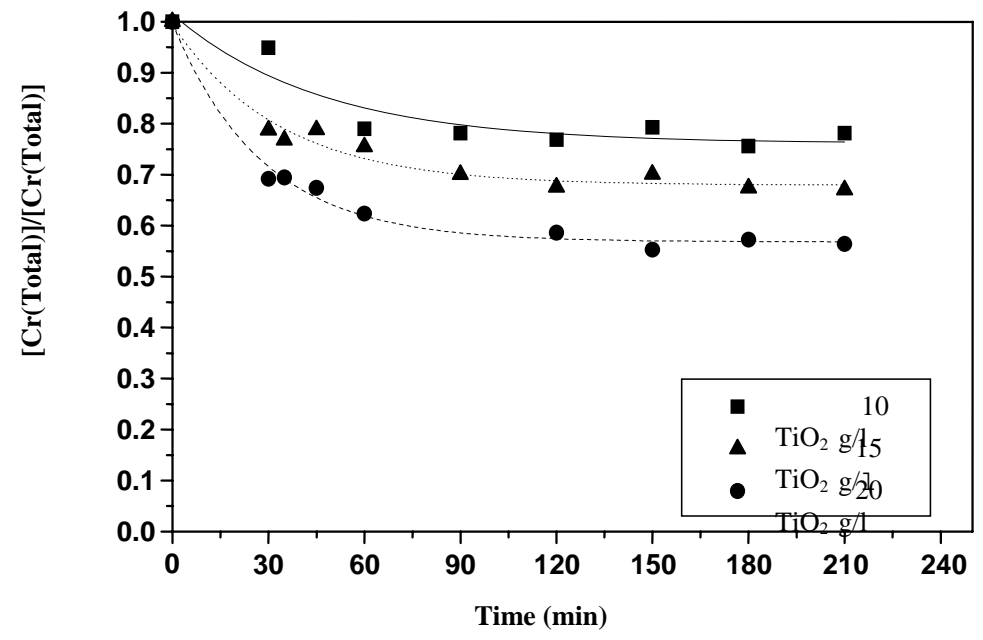

Fig. 3: Direct photoreduction of total chromium using different amounts of $\mathrm{TiO}_{2}$ 


\subsection{Indirect photoreduction of chromium on titanium using dioxide surface}

Indirect photoreduction is a photocatalysis reduction system with some addition of hole scavengers. In this study, the added hole scavenger substrate was formate ions. Results of indirect photoreduction of total chromium are shown in Fig. 4.

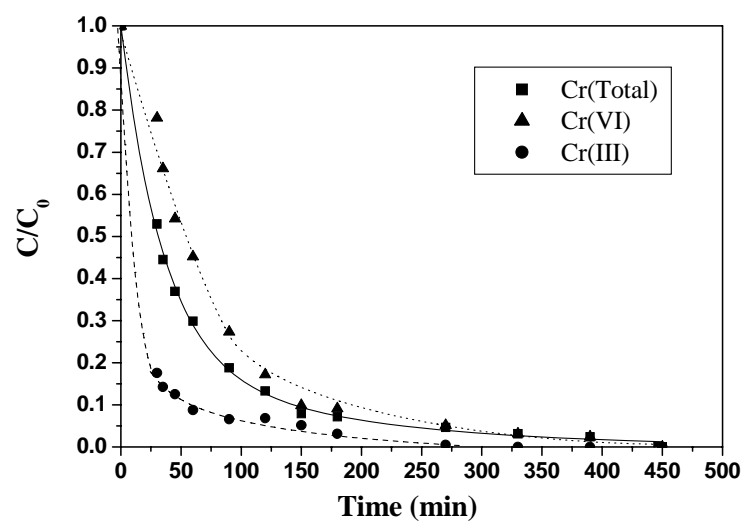

Fig. 4: Indirect photoreduction of chromium from industrial wastewater $\left([\mathrm{Cr}]_{i}=300 \mathrm{mg} / \mathrm{l}, \mathrm{pH} 3.65, \mathrm{TiO}_{2} 20 \mathrm{~g} / \mathrm{l}\right)$

It can be clearly seen that total chromium in this system was drastically reduced and even chieved higher efficiency than when using the direct photoreduction as shown in the earlier experiment. The marked increase in the percentage of total chromium photoreduced after 3 hours illumination was found to be 90 with $20 \mathrm{~g} / \mathrm{l}$ of loaded titanium dioxide. It is interesting to note that the decrease of chromium(III) mainly resulted from the adsorption process rather than the illumination process. This is because of the effect of formate ions as discussed in another study [8]. As formate ions were presented into the solution, $\mathrm{pH}$ was increased yielding the increasing chromium(III) adsorption.

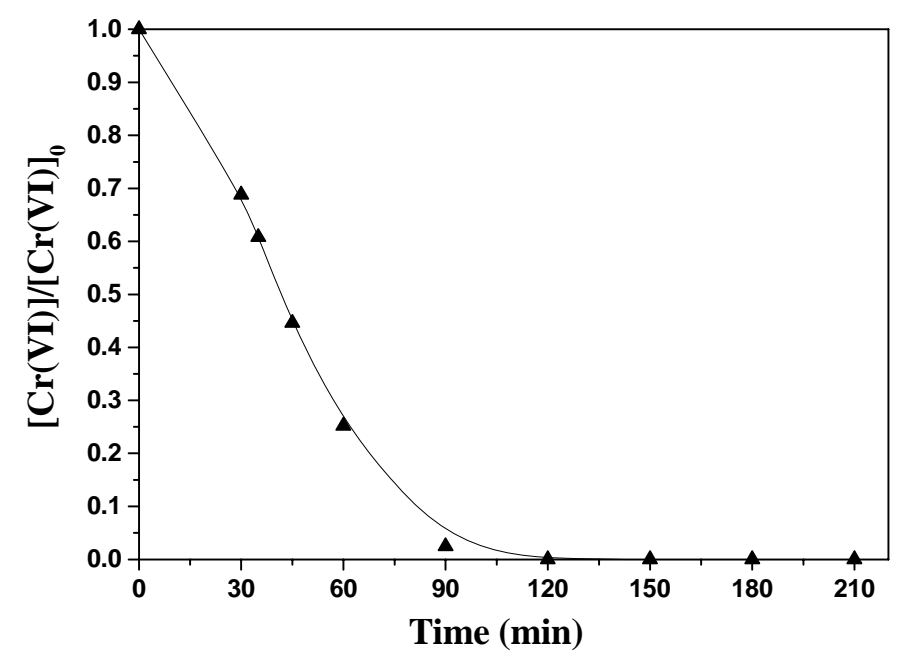

Fig. 5: $\quad$ Indirect photoreduction of chromium(VI) from synthetic wastewater $\left([\mathrm{Cr}(\mathrm{VI})]=150 \mathrm{mg} / \mathrm{l}, \mathrm{pH} 3.65, \mathrm{TiO}_{2} 20 \mathrm{~g} / \mathrm{l},\left[\mathrm{HCOO}^{-}\right]=0.1 \mathrm{M}\right)$ 


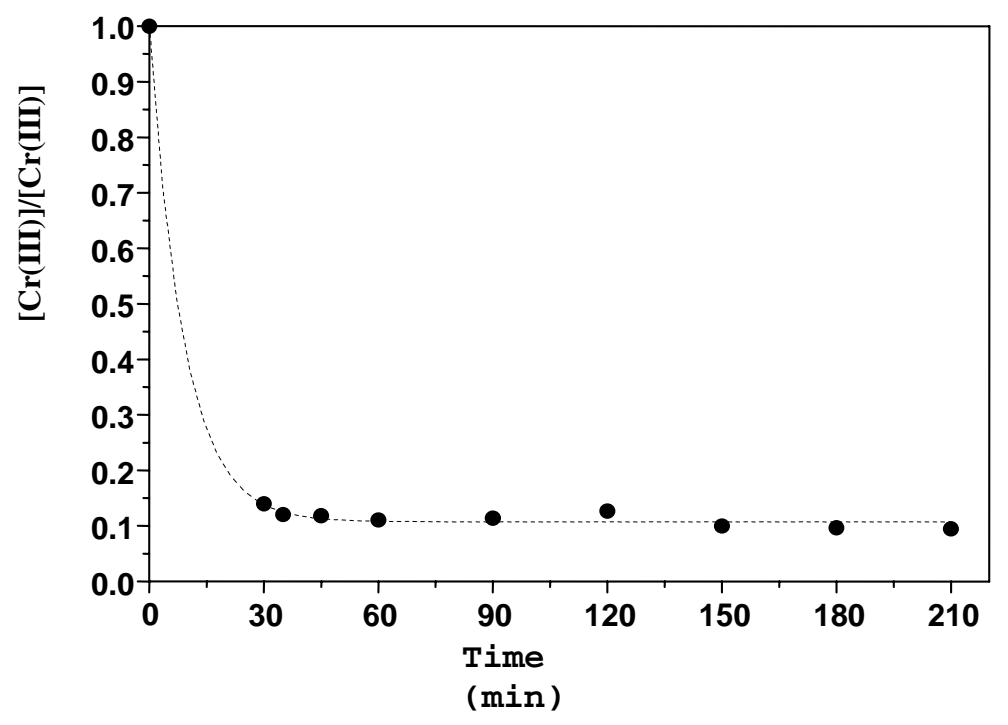

Fig. 6: $\quad$ Indirect photoreduction of chromium (III) from synthetic wastewater

$$
\left([\mathrm{Cr}(\mathrm{III})]=150 \mathrm{mg} / \mathrm{l}, \mathrm{pH} 3.65, \mathrm{TiO}_{2} 20 \mathrm{~g} / \mathrm{l},\left[\mathrm{HCOO}^{-}\right]=0.1 \mathrm{M}\right)
$$

To further explore the mechanism, indirect photoreduction of chromium(VI) and chromium(III) were performed separately. Results of photoreduction of chromium(VI) and chromium(III) are shown in Figs. 5 and 6, respectively.

Apparently, chromium(III) does not undergo photoreduction. This is because of the unsuitable redox potential of chromium(III) comparative to the potential of the conduction band edge of titanium dioxide. In contrast, the photoreduction of chromium(VI) was very favorable under these employed conditions.

Without hole scavengers (formate ions) a tentative scheme of the possible reactions for the photocatalysed reduction of chromium(VI) using titanium dioxide as a photocatalyst may be summarized in the following explanation. As the solution $\mathrm{pH}$ was less than 7 , the predominant species of chromium(VI) was $\mathrm{Cr}_{2} \mathrm{O}_{7}{ }^{2-}$. Under UV/visible illumination, chromium(VI) was photocatalytically reduced to chromium(III) by the electrons photogenerated in the titanium dioxide conduction band as shown by the following equations [7]:

$$
\begin{aligned}
\mathrm{Cr}_{2} \mathrm{O}_{7}^{2-}+\mathrm{H}_{2} \mathrm{O} & \longrightarrow 2 \mathrm{CrO}_{4}^{2-}+2 \mathrm{H}^{+} \\
\mathrm{CrO}_{4}{ }^{2-}+8 \mathrm{H}^{+}+3 \mathrm{e}^{-} & \longrightarrow \mathrm{Cr}^{3+}+4 \mathrm{H}_{2} \mathrm{O}
\end{aligned}
$$

Simultaneously, water was oxidized to oxygen by reaction with holes photogenerated in the valance band of titanium dioxide:

$$
\mathrm{H}_{2} \mathrm{O}+2 \mathrm{~h}^{+} \longrightarrow 1 / 2 \mathrm{O}_{2}+2 \mathrm{H}^{+}
$$

with these reactions, chromium(VI) can undergo direct photoreduction processing. As the titanium dioxide was restricted, the photoreduction of chromium(VI) could not approach further over the surface limitation.

In the heterogeneous photocatalytic system with hole scavengers, it is well-known that the photoreduction efficiency is improved $[3,10]$. The role of this hole scavenger addition has already been discussed in that this ion species served to intercept hole carriers which maintained the photooxidation process. With this conjugate reaction, the recombination of electron-hole 
pairs was inhibited. Presumably, the formate ions capture hole from valence bands. Accordingly, there were plenty of free electrons for chromium to react with and to create the progression of the photoreduction process.

In view of the promising results obtained when using this photocatalysis technology to remove chromium contaminant from industrial wastewater, this research finding is provide as a crucial evidence for real wastewater application.

\section{CONCLUSIONS}

The major findings of this study are as follow:

- Photoreduction of total chromium was increased with increasing amounts of loaded titanium dioxide, suggesting the dependence of the efficiency on the amount of surface sites of the catalyst.

- The indirect photoreduction yielded a higher efficiency in chromium removal than the direct process. This finding shows the important role of adding hole scavengers to prohibit the electron-hole pair recombination effect.

- This employed technology efficiently removed all species of chromium (total, hexavalent and trivalent chromiums) from chrome-elecroplating wastewater.

\section{ACKNOWLEDGEMENT}

We are grateful to the National Metal and Materials Technology Center, National Science and Technology Development Agency, Thailand for financially supporting to this work.

\section{REFERENCES}

1. Chen, D. and Ray, A.K. (2001), Removal of Toxic Metal Ions from Wastewater by Semiconductor Photocatalysis, Chemical Engineering Science, vol. 56, pp. 1561-1570.

2. Chenthamarakshan, C.R., Rajeshwar, K., and Wolfrum, E.J. (2000), Heterogeneous Photocatalytic Reductin of Cr(VI) in UV-Irradiated Titania Suspensions: Effect of Protons, Ammonium Ions, and Other Interfacial Aspects, Langmuir, vol. 16, pp. 2715-2721.

3. Deng, B. and Stone, A.T. (1996), Surace-Catalyzed Chromium(VI) Reduction: $\mathrm{The}^{\mathrm{TiO}} \mathrm{2}^{-}$ $\mathrm{Cr}^{\mathrm{VI}}$-Mandelic Acid System, Environmental Science and Technology, vol. 30, pp. 463-472.

4. Kajitvichyanukul, P., Chenthamarakshan, C.R., Qasim, S.R., and Rajeshwar, K. (2002), Photocatalytic Reactivity of Thallium(I) species in Aqueous Suspensions of Titania, Journal of Electroanalytical Chemistry, pp. 519, 25-32.

5. Kajitvichyanukul, P. and Vatcharenwong, A. (2004), Influence of pH, Organic and Inorganic Anions on Photocatalytic Reduction of Chromium(VI) With Titania Powders, Asean Journal of Science and Technology for Development (submitted).

6. Kajitvichyanukul, P., Taweekitwanich, A., Toyam N., and Pongpom, S. (2004), Effect of Organic and Inorganic Ions on Chromium(VI) Adsorption onto $\mathrm{TiO}_{2}$ Surface, Asean Journal on Science and Technology for Development, vol. 21, pp. 249-258. 
7. Khalil, L.B., Mourad, W.E., and Rophael, M.W. (1998), Photocatalytic Reduction of Environmental Pollutant Cr(VI) over Some Semiconductors under UV/Visible Light Illumination, Applied Catalysis B: Environmental, vol. 17, pp. 267-273.

8. Ku, Y. and Jung, I-L. (2001), Photocatalytic Reduction of Cr(VI) in Aqueous Solutions by UV Irradiation With the Presence of Titanium Dioxide, Water Research, vol. 35, pp. 135142.

9. Lin, W.-Y., Wei, C., and Rajeshwar, K. (1993), Photocatalytic Reduction and Immobilization of Hexavalent Chromium at Titanium Dioxide in Aqueous Basic Media, Journal of Electrochemistry Society, vol. 140, pp. 2477-2482.

10. Litter, M.I. (1999), Heterogeneous Photocatalysis Transition Metal Ions in Photocatalytic Systems, Applied Catalysis, vol. 23, pp. 89-114.

11. Rajeshwar, K. and Ibanez, J.G. (1997), Environmental Electrochemistry, Academic Press, San Diego, USA. 